\title{
A Model of Achieving Safe Interoperability of Medical Data in the Private Sector of Health Care in Romania
}

\author{
Madalina-Elena RAC-ALBU ${ }^{1}$, Marius RAC-ALBU ${ }^{2}$ \\ 1, 2 "Carol Davila" University of Medicine and Pharmacy Bucharest, Romania \\ ${ }^{1}$ Bucharest University of Economic Studies, Romania \\ racalbu@gmail.com
}

The lack of interoperability in the private sector of medical services in Romania represents a big gap in completing a medical file for a patient who uses both private and public medical services. In this article is presented a model of an interoperable medical system in private medical services. This system has standardized medical documents (in conceptions) and interoperable medical documents (using documents interoperability standards). These are two different items, the first ones are referring to gathering the same medical records for all the patients for every medical field (e.g.: cardiology, dermatology etc.) and the second ones are referring to transport standards like HL7/CDA (Health Level 7/Digital Imaging and Communications in Medicine). This new designed system has a big focus on protecting the personal data of the patients by respecting the European Law about personal data sharing (General Data Protection Regulation-GDPR) specially article no. 26 which requires pseudonymization and anonymization of personal da and medical records. [1]

Keywords: Interoperability of Medical Data, HL7/CDA, EHR (Electronic Health Record), IT Medical System, Protection Of Medical Data

\section{1} Introduction

Currently Romania benefits of a medical IT system in the public sector of medical providers. This system is implemented but is not totally functional. The system is called SIUI (Sistemul Informatic Unic Integrat - The Unique Integrated Informatic System) and has three points of action: Health Card, Electronic Prescription and Medical File or EHR of the patient. We have presented the Romanian EHR situation in the article "Interoperability of Medical Data Through e-Health Service in Romania". [2]

In the private sector of health care providers in Romania there is a total lack of interoperability of medical data although the private medical sector has grown tremendously in the last years. According to the Romanian National Institute of Statistics, the private medical system has expanded 60 times since 1997, so that private medical infrastructure has now reached about $75 \%$ of all medical units in Romania.

This development of the private health system has been achieved especially in the urban environment and it is mainly related to private medical practices. Although the number of private hospitals has increased considerably (from 2 in 1997 to 161 in 2016), however 160.000 patients have been hospitalized in one year, representing only $4 \%$ of the total hospitalized patients. But even with this high rate of expanding of private hospitals, they now have just 6.600 beds and the public hospitals have 125.000 beds and a plus for the public hospitals is the high multidisciplinary which is not achieved yet in all private hospitals. This creates the premises for an enormous potential increase in hospital units in the private system, which means that we are in the expanding private health sphere. In this context it is very important to achieve the interoperability of medical data from the private health system.

Medical data represents the totality of medical information that we can gather for one patient in a unit of time or for all his life. When we refer to medical data that are gathered, managed and visualized by just a medical unit and refers to just an episode of health care problem for one patient, we call it EMR Electronic Medical Record. When we speak of medical data that use the interoperability standards and can be gathered, managed and 
visualized at national or international level and is not observing a single medical episode, but a life time history of medical data we call it HER - Electronic Health Record. [3]

The existing of EHR is mandatory in order to obtain interoperability of medical data but it is not enough. For example, in the USA over the last years it was a tremendous action at national level in order to complete the EHR data without taking in consideration the next step of connecting the EHR's in order to achieve interoperability. [4]

The interoperability of medical data is a very important goal for all countries of European Union and not only because in nowadays, when the mobility of people is increasing and every person can work or live in any European country, it is absolutely necessary to have their medical records ready to be used wherever they are living or working, nonmatter if is in Romania or other European country.

A definition of interoperability given by HIMSS (Healthcare Information and Management System Society) explains very well what represents this concept and how it is to be achieved. "In healthcare, interoperability is the ability of different information technology systems and software applications to communicate, exchange data and use the information that has been exchanged. Data exchange schema and standards should permit data to be shared across clinician, lab, hospital, pharmacy, and patient regardless of the application or application vendor." [5] For a system to be interoperable it is mandatory for the documents that make the object of this interoperable system to respect the same structure, form and components.

The most important question that has to be answered is how to obtain medical data that can be interoperable to all medical systems. The answer is STANDARDIZATION.

Standardization represents the key to achieve correct interoperability, but standardization must be considered by its two points of view: a) The standardization of the way the clinical data are gathered - Clinical Data Standard CDS. In all medical fields the doctors create a standard questions protocol in order to establish the diagnosis of a disease. But not all the doctors have the same protocol of investigating a patient, therefore the medical data gathered and stocked in a personal medical file for a patient could be different from a medical service provider to another. In order to achieve interoperability, it is a must to have the same structure of the way the data are collected. All the medical organizations have understood that prerogative and now it is being developed a general effort in obtaining common questioners that should be used by all the doctors which are acting in the same medical field having the final aim to obtain a standardized clinical data. For example, in cardiology the National Cardiovascular Research Infrastructure (NCRI) is a project that has the final aim to create a model for standard data exchange, clinical registry including all EHR. This project was initiated by Duke Clinical Research Institute and American College of Cardiology. This project started in 2009 and the finally aim of it is to create a common vocabulary for cardiology, a cardiovascular vocabulary that should be the first base for creating syntactic and semantic interoperability. There are identified 99 key terms that can used in every cardiovascular subspecialty in every general-purpose EHR. [6]

American College of Cardiology Foundation (ACCF) and the American Heart Association (AHA) are united with the only direct purpose to create a benefic environment in order to prevent cardiac disease, the optimization of cardiovascular research and a better way to diagnose and treat a patient. Therefore, they recognize the importance of Clinical Data Standardization (CDS) and they continuously carry this activity of extending the portfolio in order to create new clinical data standard. CDS represents in fact common data element which is the base of obtaining semantic interoperability. [7]

Data standardization can only exist if there is effective coding. Codes are the key to standardization, as they can make it possible for data to be interpreted correctly by other users, who have direct access to data fields. 
Text data, which is not coded (words, phrases or paragraphs) prevents interoperability. In the medical field, there is a huge number of diseases and diagnoses, treatments and medications. These have been structured and classified according to the medical field they are referring to. Known classification systems are:

ICD-10 (International Classification of Diseases - 10th Review), ICPC-2 (International Classification of Primary Care, Second edition), DRG (Diagnostic Related Groups), SNOMED (Systematized Nomenclature of Medicine-Clinical Terms) etc.

According to the National Alliance for Health and Information Technology (NAHIT), it is very important to automatically transfer complementary diagnostic procedures for electronic medical record systems that should be accessible through other computer systems without the need for additional translation or semantics translation.

b) Interoperability between health information systems is only possible through the definition of standard messages, which must be adopted by all manufacturers of this type of technology in order to make the functioning of these systems efficient. [8]

Among the different standards used, they should be highlighted by their international usage: HL7 (Health Level Seven) and DICOM (Digital Imaging and Communication in Medicine) [9]

The most used method of creating or using medical data is using HL7 messages. "This "common language" allows healthcare organizations to integrate different applications with the support of existing IT environment in the organization." [10]

HL7/CDA (Clinical Document Architecture) is a model for sharing medical records. CDA converts documents that can be read both by computer and human operators, using the XML standard [11] The CDA allows clinical documents to be compatible with the medical informatics field using XML, HL7 RIM (Reference Information Model) and medical computer codes and also attempts to maintain a high level of understanding and assimilation by medical staff. Content of CDA documents can be viewed using web browsers (due to XML based).

DICOM is the standard for communicating and managing medical imaging information and related data. The DICOM standard covers the field of medical informatics. Within this domain, it addresses the exchange of digital information between medical imaging equipment and other systems. Because such equipment can interoperate with other medical devices, the purpose of this standard should overlap with other areas of medical informatics [12]. The most important problem that should be correctly and well managed is the ensuring confidentiality and privacy of patient's medical records.

\section{Defining a Medical Interoperable IT System in the Private Health Services}

Medical data must have a basic feature without this being not possible to have interoperability: CONFIDENTIALITY. The patient whose medical data must be transferred between different computer systems is the one who has to give his consent to this. So, the interoperability of medical data between different computer systems can only be achieved with the consent of the patient. Therefore, it is very important to define how the patient agrees, and more clearly, the way to identify and certify the patient.

\subsection{Explaining the Model of this System}

The proposed system is using all the standards described below in order to obtain interoperability like all the interoperable medical systems, but the main focus of this system is on protecting the medical data of the patient, which is very important in nowdays. The system has in his center an authority, called Central Authority (CA) that will represent the most important entity which will certify the existence of the patient and the existence of all medical providers. This interoperability authority will be the secret keeper of all information that is necessary to identify a person or a medical care provider.

A patient has a unique digital identity for his 
identification in $\mathrm{CA}$ and a different digital identity for each medical cabinet. Digital CA identification is generated by identifying the patient with his/her personal data, representing his or her identity in CA.

The digital identity for each medical clinic is granted by the CA, each cabinet knowing only the identity of the respective cabinet. Thus, the patient has ID1-CAB1, ID2-CAB2, ... n-CAB n.

A patient is presented to the medical clinic $X$ for consultation with a cardiologist for the condition he or she suffers. The physician wishes to visualize recent cardiac ultrasound in another medical clinic, as well as the patient history of all cardiac investigations of the patient's cardiac affection in order to establish a correct, evolving diagnosis and adapt the treatment to current disease changes.

When the physician in $\mathrm{CAB} 1$ decides that he needs the patient's medical data from other clinics (as in our example) sends to CA a request in which there must be specified the patient's ID in CAB1 and the medical data he or she needs. This application will have a number by based on which it will be identified in all the computer systems in where it appears. The CA, after receiving the request, will also request the patient's consent to the data transfer. It may request and receive the patient's consent in a mutually agreed manner (phone messages, token devices, token on the mobile phone etc.). With the patient's consent $\mathrm{CA}$ sends to $\mathrm{CAB} 1$ a token code announcing CAB1 which will be the method of logging in to the system for obtaining the data. CA will spread this request to all medical units where the patient's ID appears, obviously only CA knowing all these different IDs for each cabinet.

Currently, in Romania none of the private medical providers are not connected in no way to each other or connected to public healthcare system at all. It is absolutely necessary to have a system designed to be interoperable in order to create a very successful and benefic environment in the Romanian medical care private system.

\section{Participants or Actors of the Proposed System}

Central Authority may be an authority that has access to the patient's personal data and can authenticate and authorize both the patient and all participants in the transfer of medical data. It will be a TRUSTED authority recognized and agreed upon by all participants in this system. The CA must comply with the following functional specifications (FS):

1. must be able to uniquely identify a patient using digital identity

2. confers uniqueness and anonymity on every patient, and its medical data is confidential

3. the authority does not have access to medical data, its purpose being to provide authentication and authorization services for computer systems that will effectively transfer medical data

4. provides digital identities to all medical units in the same way as they offer to patients

5. it is designed to create a system whereby any medical data transfer is done anonymously so it is impossible for the clinic that owns the medical data to identify the patient for whom data transfer was requested

6. must provide mechanisms by which a patient can accept or refuse any transfer of his / her medical data

7. receives the request from medical clinic $\mathrm{X}$ that already has a digital identity

8. interrogates the patient and asks for his consent

9. upon agreement with the patient, he agrees with $\mathrm{X}$ clinic to receive data from the clinics holding the required data (the patient decides whether the $\mathrm{X}$ clinic can receive data or not)

Patients are all persons who address at any moment to any Medical Unit (MU) acting in the private health system in Romania (medical analysis office, hospital, medical office or Para clinical investigation center). Patients will be assigned a unique identifier in a system called Digital Identity (ID1) through which they will be identified in CA. Digital identity does not contain medical data. 
Patients will also have a different identity in each MU they call at one time, namely ID $1,2,3, \ldots \ldots, \mathrm{n}$ represented by the identities specific to each MU, consisting of several identities, so that the medical units cannot identify the patient after this identity

Patients must give their consent of any transfer of medical data from the system. Without their consent, no transfer of medical data is possible. If the patient is not able to give his consent about his medical data transfer, a person in his family or not, chosen by the patient will perform the agreement of medical data transfer.

Medical Units represents all establishments where private health services are offered. These may be: medical cabinets of specialist doctors, private hospitals, blood sampling centers, Para clinical investigation centers, etc. MUs will also have a unique digital ID assigned by an AC called IDUM that can be obtained when enrolling in this system.

\subsection{Data Types Existing In This System}

Digital IDs - are those data whereby each participant in the system is assigned a unique identity by $\mathrm{CA}$ based on identification data provided by Central Authority participants. IDs are obtained for the two categories of participants as follows:

A. for patients - ID - they provide the CA all the necessary data for a correct identification of a patient such as: Name, First Name, Identification Number, Address, Phone, Series and CI Number, etc.

B. for medical units - IDUM - they provide the CA with all the data that they deem necessary for a correct identification of each MU such as: Name, Tax ID or ID assigned by the College of Physicians, Headquarters Address and Points Existing Work, Phones, etc.

Medical data - MD - are those data that contain medical information related to a patient that will be transferred to this system, their safe and prompt transfer being the main objective of creating this system. They may be documents or non-documents (images, signals, etc.); documents must be in the same format for all participating MUs and nondocumentary documents generated by MRI, paraclinical investigations. MRI information (images) are automatically generated according to DICOM standard. Medical data are specific to each medical specialty and must be clearly identified for the transfer.

System data - SD - these are data used in Business Logic processes: data transport, security, audit, etc. System Data may be for example medical data requested by a medical unit for a particular patient. Each application will have a unique identifier that will be recognized by all participating healthcare units. This request number will be assigned by the $\mathrm{CA}$ at the time the request is made and reaches it. System Data may also may be the response to the existence or non-existence of medical data in the medical units where the request is launched.

\section{Architecture of an Information Transfer System}

The obtaining of an interoperable IT system in the private health sector has a unique purpose: secured transfer of all types of data among all participants in this system.

Data transfer within the system between any two entities participating in the system is performed using machine-machine-specific communication technologies. From a software point of view data transfer is performed using client-server (Web services) technologies. In a Web service, Web technology, such as HTTP initially designed for person-tomachine communication is used to achieve machine to machine communication, more precisely for transferring file formats such as XML and JSON (Java Script Object Notation).

XML or JSON files are ways to encapsulate the data to be transferred to the system. For example, in the proposed information system, the transfer of information follows the path as follows:

Every medical unit in the system that holds the required medical data will automatically transfer the data, so MU1 will receive the medical data needed to make the medical decision. Of course, medical data may be 
required for a certain amount of time depending on the needs of the treating physician.

The most important aspect of this system is the specificity of the medical data asked for. For example, a cardiologist needs a very specific type of medical data gathered in a specific unit of time in order to take the best medical decision, he doesn't need to consult all medical data that are gathered in the personal medical file of the patient. There are also other data that are being transferred to the system.

The architecture of the proposed system is based on Central Authority, which has the most important role. The other participants to the system can become participants only after they register to $\mathrm{CA}$. The communication between Medical Units and CA and patient and $\mathrm{CA}$ is described in Figure 1.

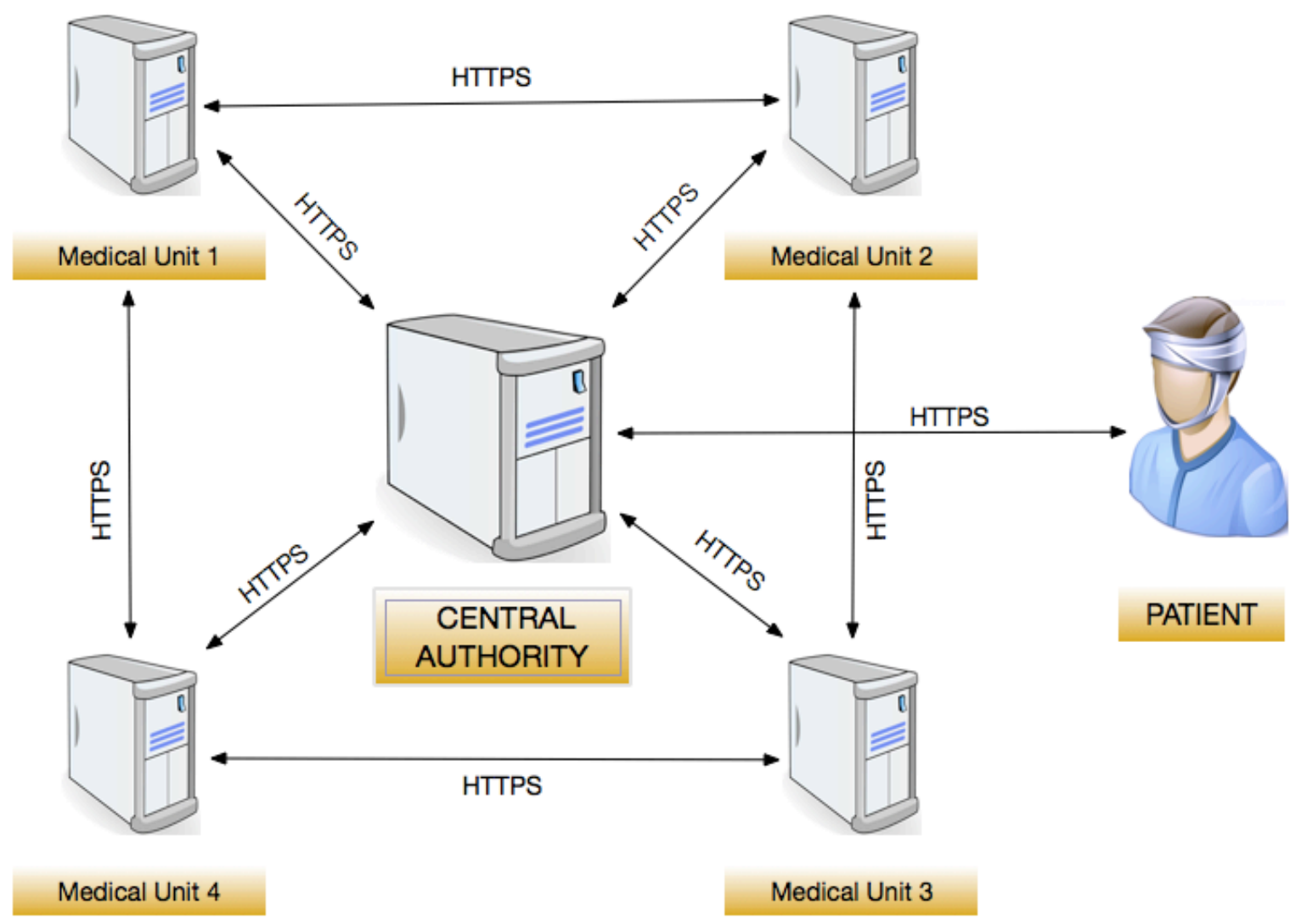

Fig. 1. Architecture of the proposed medical system

Other important aspect of data communication between two computer systems is security. Within the proposed solution, two ways of assuring it are distinguished:

A. Encryption using Hyper Text Transfer Protocol / Secure (HTTPS) Data Protocol - As can be seen in Figure 1, the data transfer will be done using HTTPS, which is a communication protocol designed to transfer encrypted information over the WWW. This protocol protects the data during the transfer using one or two
Secure Sockets Layer or TLS (Transport Layer Security) protocols. The launch of an HTTPS connection involves first sending the SSL certificate and then transferring the data effectively. HTTPS is therefore a method of authenticating the server that uses it via the digital certificate, the browser requires the server to start the encrypted transfer. Digital certificates are issued by authorities recognized by both parties to the exchange so that the browser can be sure the server with which it communicates is 
the one that claims to be. Thus, in the proposed IT system, each MU will need to acquire a digital certificate that is recognized by all participants in the system. It is the Central Authority that will make the selection of the digital certificate issuers approved throughout the system, thus making it possible to transfer data through HTTPS.

B. The second method is data anonymization: - All data transmitted is anonymous. If someone intercepts documents transferred to the system, they cannot use it because it is impossible to find out who owns the specific medical data. The Central Authority provides each patient with an ID based on CA identification and a Single Code that identifies the patient in the system.
Patient identification data are exclusively administered by CA. The patient is presenting himself to the MU, where he will be identified by the Unique Code given by the CA, all the patient's medical data resulting from the consultation will be stored in the MU database where the consultation is performed but will be stored anonymously. The patient will receive an ID from the authority, an ID that will not be known to the physician, so that no one can view the patient's medical data without his consent.

5 The Prototype of an Actor in the Information Transfer System

Each actor consists of an application with a specific software architecture. This architecture is presented in Figure 2.

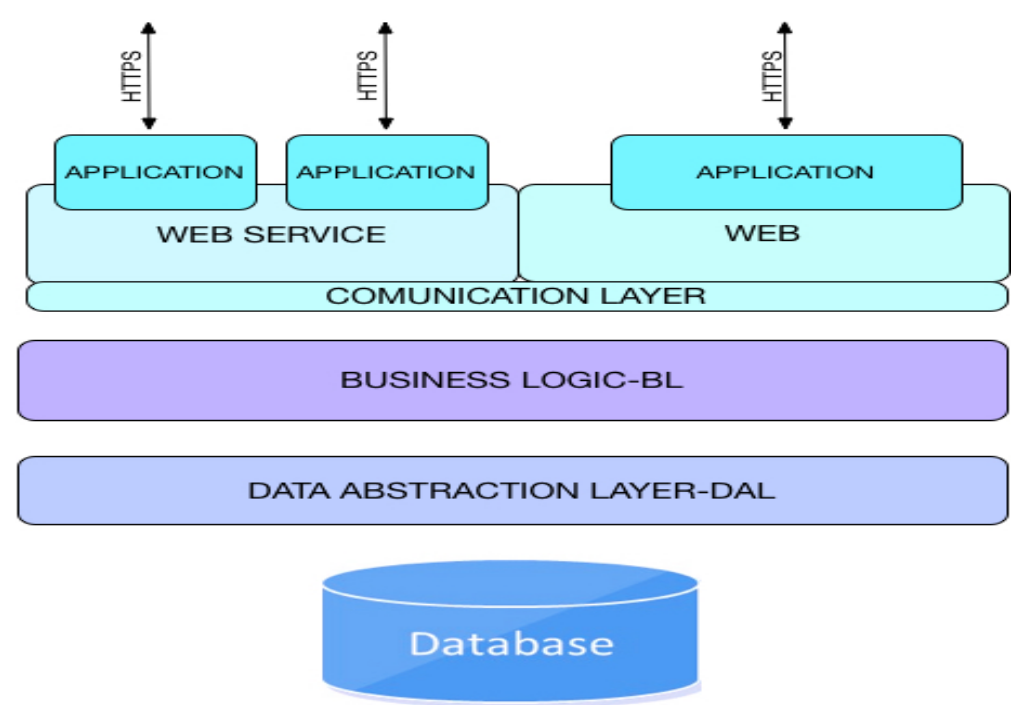

Fig. 2. Software architecture for every actor of the system

DAL = Data abstraction layer - represents the data model and the mechanisms for saving the information in DB. It is an application programming interface that unifies communication between a computer application and databases such as SQL Server, DB2, MySQL, PostgreSQL, Oracle or SQLite.

BL = Business Logic - consists of a BI framework that executes streams based on the data received from the communication. In software BL is the part of the program that encodes real world business rules that determine how data can be created, stored, and modified. It is in contrast to the rest of the software that may be concerned about the lower level of database management or display of the user interface, system infrastructure, or generally connecting different parts of the program.

$\mathbf{C L}=\mathbf{C o m m u n i c a t i o n}$ layer - is the level at which the direct relationship is conducted.

Communication is 2 ways:

1) $\mathrm{M} 2 \mathrm{M}$ (Machine to Machine $)=$ web service

2) H2M (Human Machine) = web application

\section{Information Flows in the System}

6.1. F1-the flow of system participants 
identification can be seen in Figure 3.

The Central Authority is the authority that can give identity to each participant in the system. There are several flows through which the participants will obtain a specific identity as follows:

- Stage 1 - The patient wishing to be part of this system sends an ID request to the CA which must obtain all his / her personal data (data specified by CA)

- Stage 2 - The CA receives and analyzes the data, establishes a way of "communicating" with the patient through which it can quickly and safely receive the patient's consent regarding the medical data that belongs to the patient and then assigns an ID (ID1), which is used to identify the patient in CA

- Stage 3 - The medical unit enrolling in the system will also make an application for CA identity assignment including all its identification data, data specifically identified by $\mathrm{CA}$

- Stage 4 - The CA receives and analyzes the data and assigns a unique identity to IDUM, as well as a specific identity for each patient for every medical unit different from ID1(which is used just for identify the patient in CA)

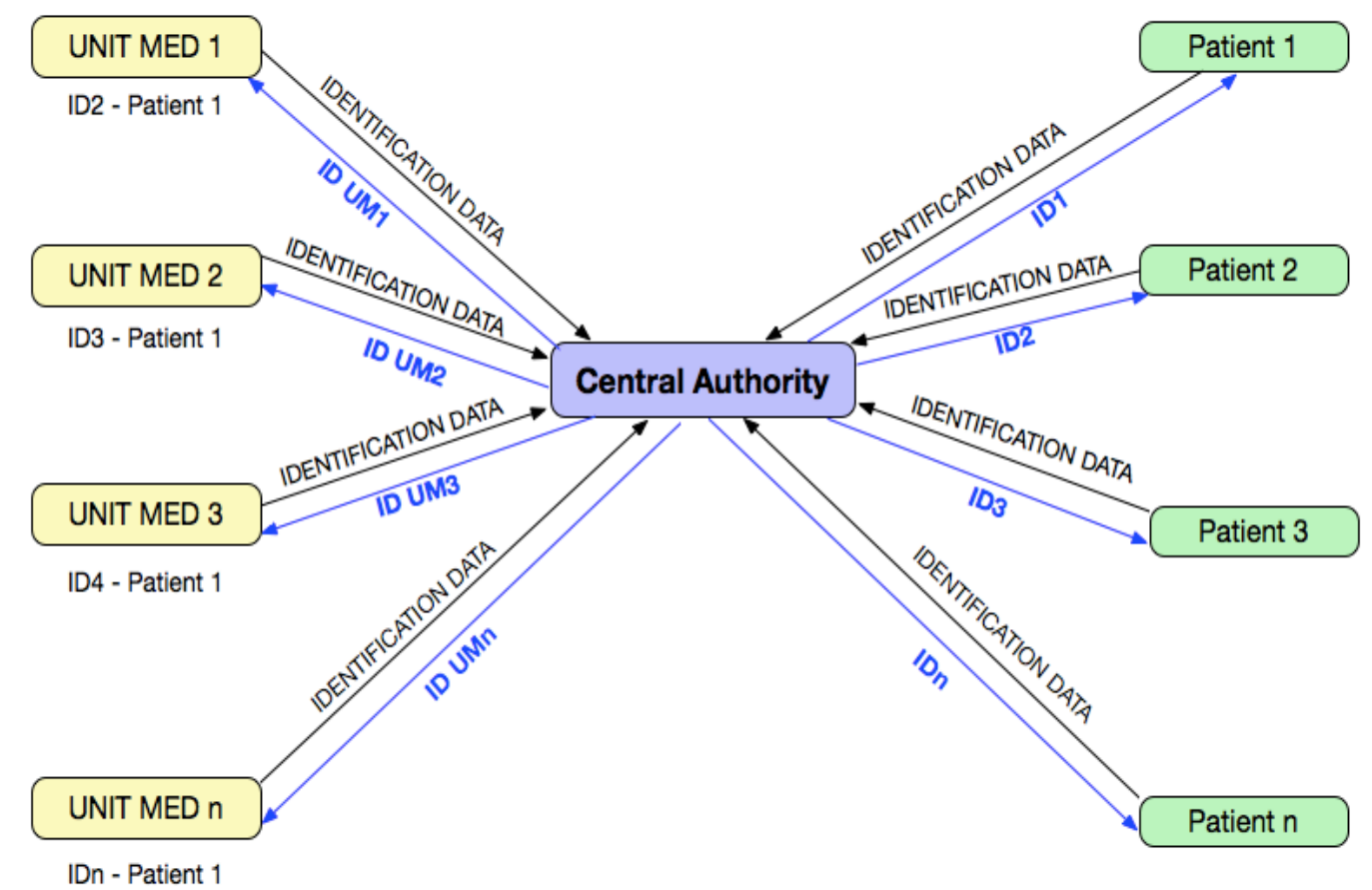

Fig. 3. Identification Flux

6.2. F2-the flow of medical data transfer can be seen in Figure 4.

Medical data is the subject of the transfer in order to achieve interoperability. It is very important that a treating physician of a patient can have access to any medical information he or she is instantly required to take the best therapeutic decision for his patient. But this transfer process must meet two major conditions:

- to be carried out in maximum safety

- to be done only with the patient's consent For this we have exemplified a first scenario for achieving this transfer presented in the following illustration, in which all 5 flows generated by the transfer are presented below:

- Step 1 - MU1 is the medical unit that needs a patient's data. It launches the application with no. 6000 (example). The request specifies the patient ID for which the request is made and the required medical data (e.g., cardiac data: keg, eco cord, Holter tension results, etc.). These will be called Specific Medical Data (SMD).

- Step 2 - CA requires the patient's consent to the transfer of this medical data into the system 
- Step 3 - The patient agrees, and the CA communicates this to the MU that made the request

- Step 4 - Upon receipt of the patient's agreement, the CA launches a broadcast message to all medical units in the system asking for the medical data that medical unit 1 needs

- Step 5 - Medical units will send the medical data of that patient to the medical unit that launched the application

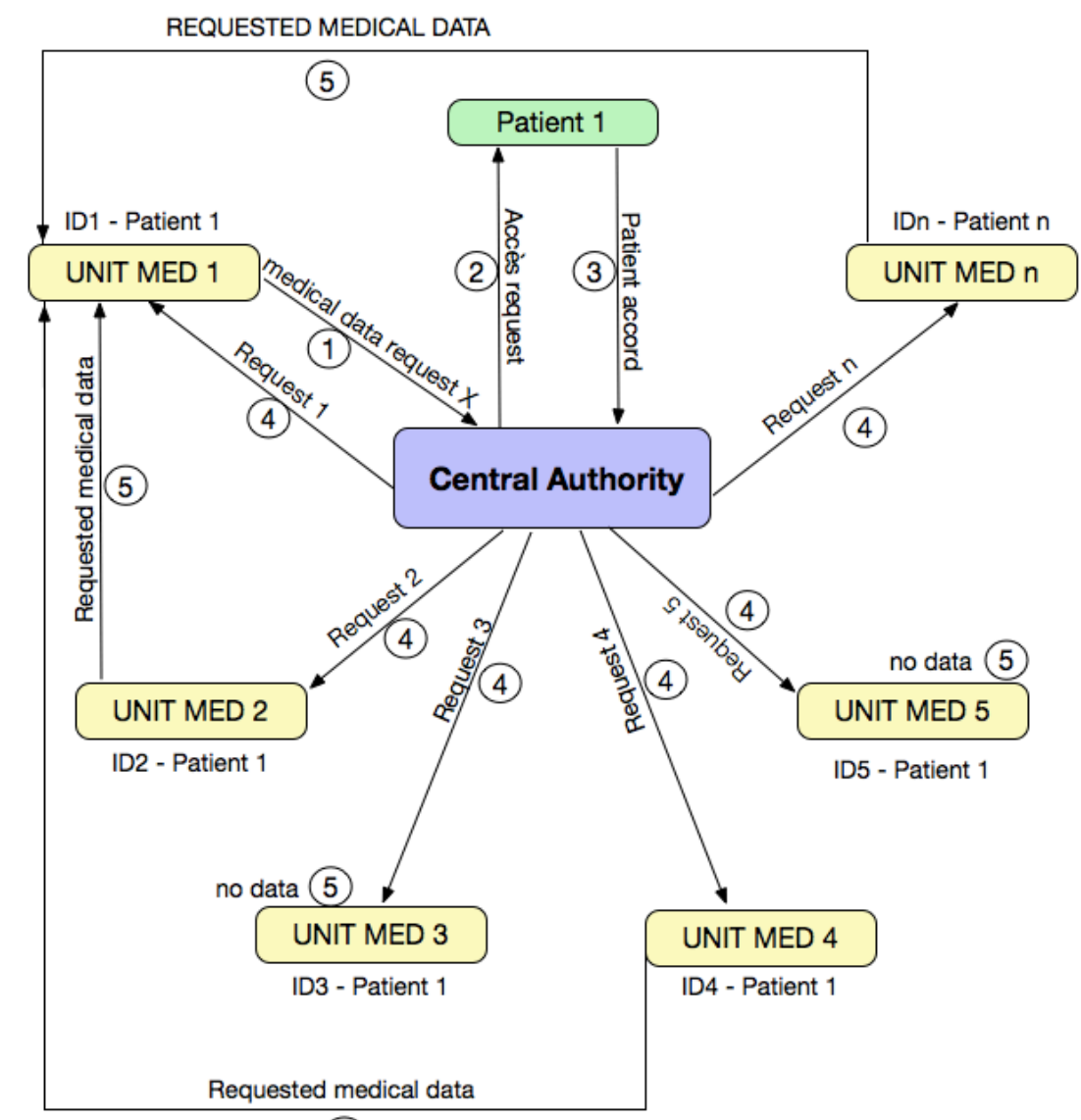

(5)

Fig. 4. Data flux

7 Business Process Model and Notation (BPMN)

"Business Process Modeling Notation (BPMN) is a flow chart method that models the steps of a planned business process from end to end. A key to Business Process Management, it visually depicts a detailed sequence of business activities and information flows needed to complete a process." [13]

Achieving BPMN creates the premises of a strategic thinking in which the flows created to achieve an objective are correctly explained.

For the flow of patient enrollment in the system described in Figure 3 it is corresponding BPMN schemes presented in Figure 5 and Figure 6. 


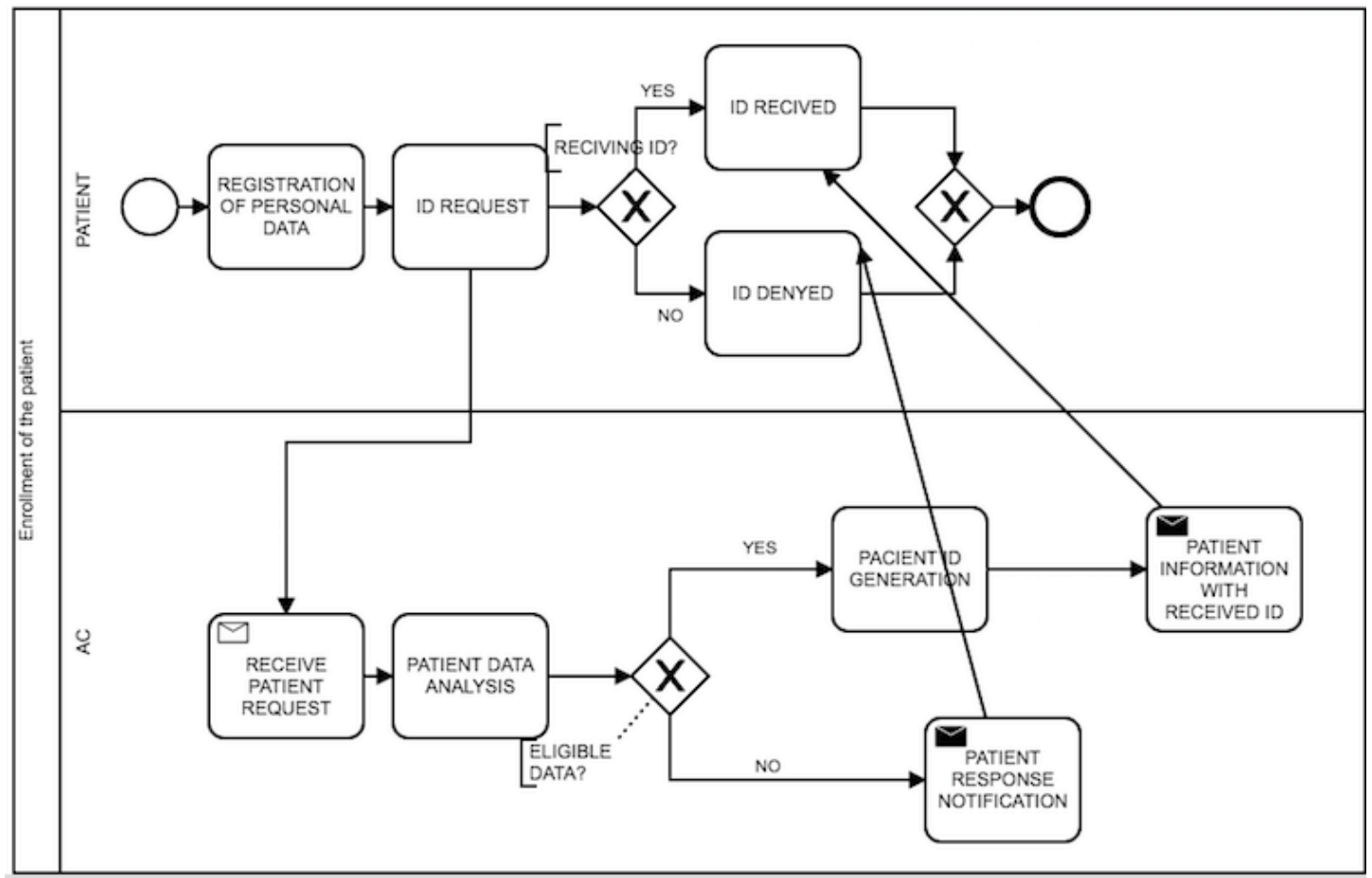

Fig. 5. Enrolment of the patient

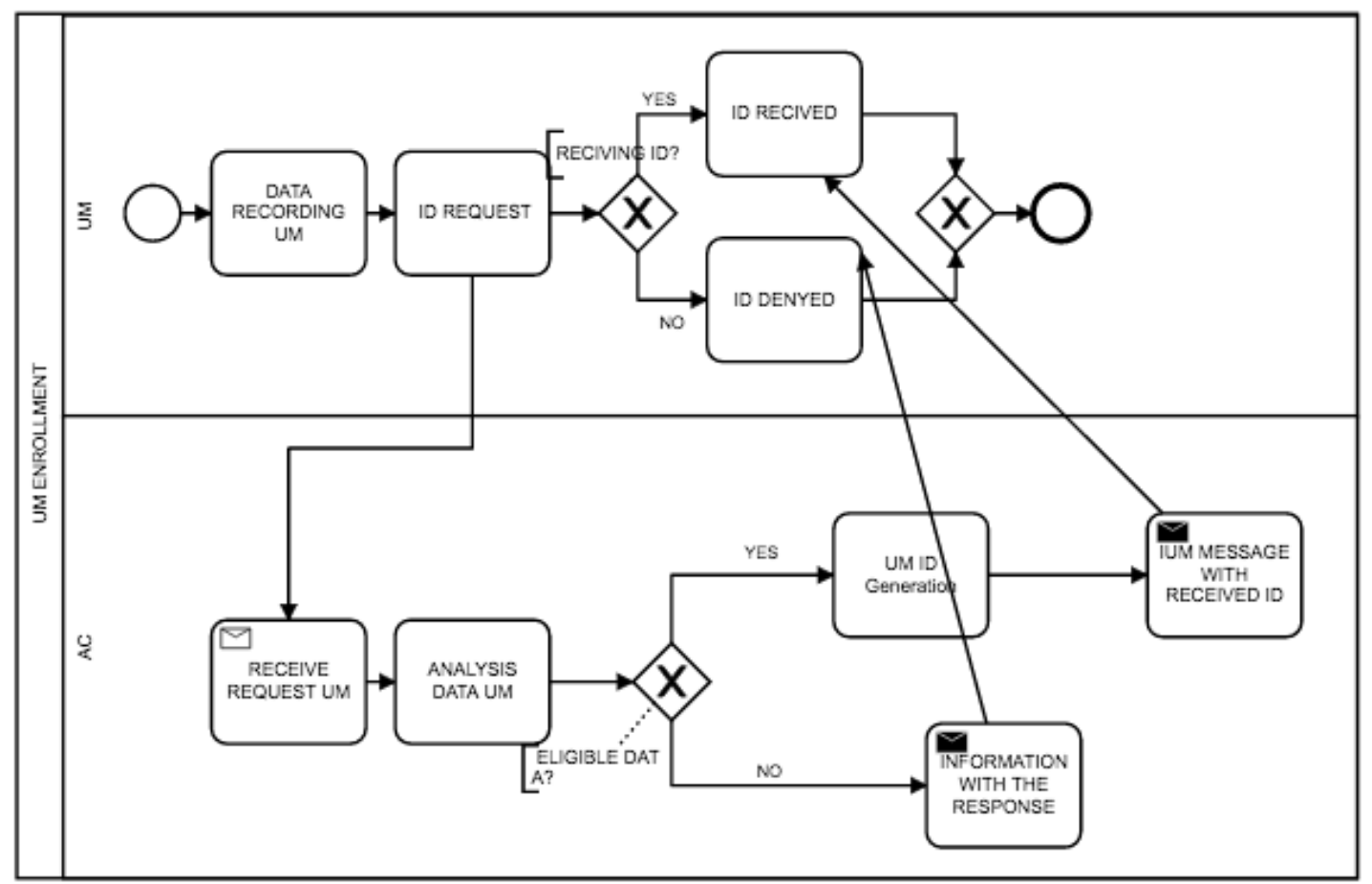

Fig. 6. Enrolment of a Medical Unit

For the flow presented in Figure 4 the BPMN system.

schema from Figure 7 is just a part of the flow, The other BPMN schemas are more complex the flow that allows the Medical Unit to but they follow the same principle. register medical data for a patient in the 


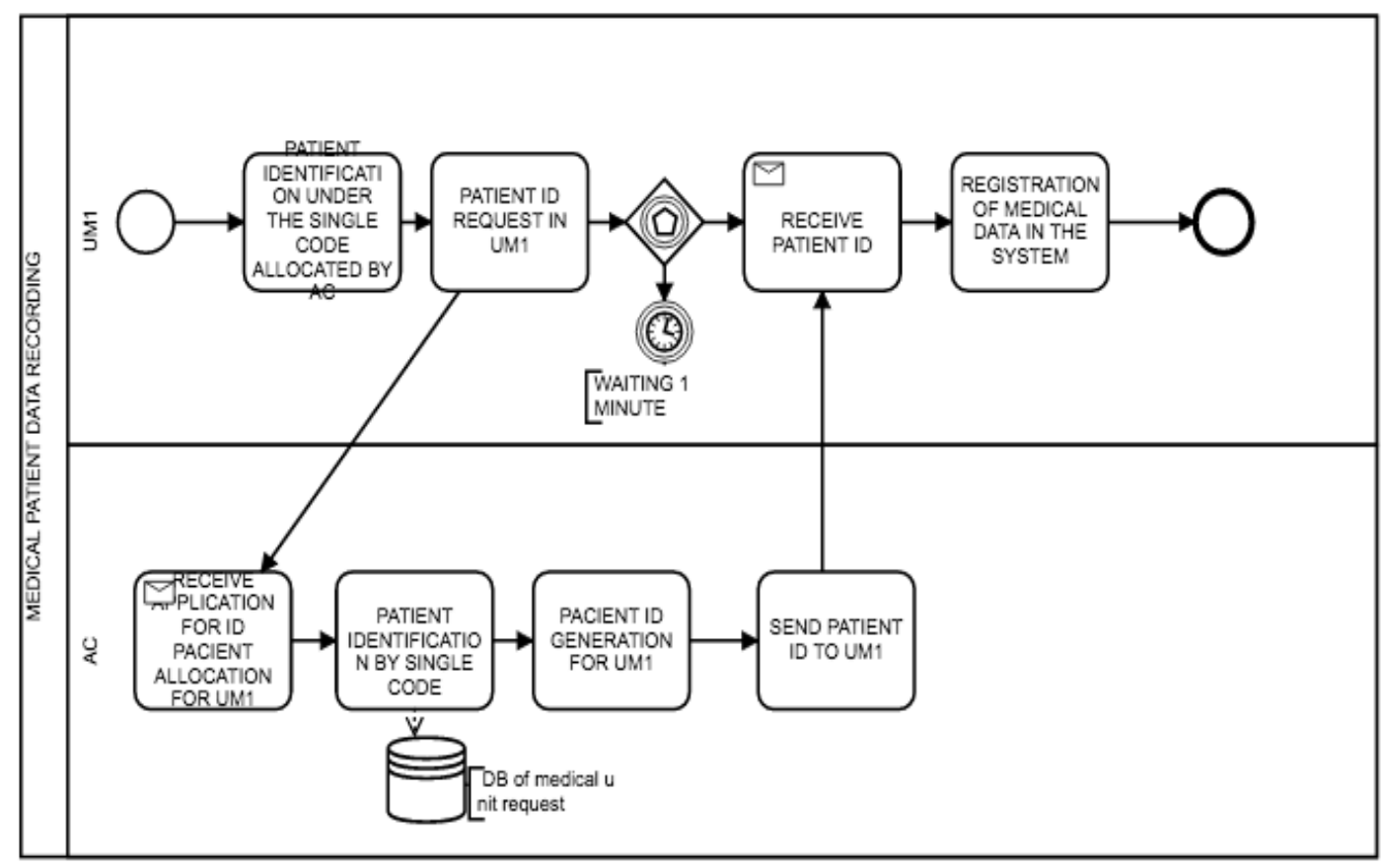

Fig. 7. Recording medical data in the system

This system has the ability of transforming a non-communicative medical system into a new one system which has the ability of interchange medical documents.

\section{Conclusion}

This article describes a vision of an interoperable system where the personal data and medical data are protected from the beginning by the design of this system. The security and confidentiality standards are use in this system in order to create a secure transport of medical data. This system has many advantages such as:

-It uses the same medical data gathered by the same structure, which means that the doctor who see the patient has a standardized questionnaire of consulting the patient and this fact reduces the chance of forgetting information that are necessary in order to put the right diagnosis

-The medical data are stored in the medical unit where they are gathered. This has the advantage of not duplicate the medical data. All the medical data can be visualized by all the participants (that are in titled) of the system (doctors), but the information "disappears ", after is used, it still remains in the primary data base where can be used again in the future.
-This system permits very easy to perform Artificial Intelligence (BI-Busies Intelligence) in order to make intensive research about the population state of health, or make prediction about any pathological field that present an interest in a period of time.

-It has the ability of reducing the costs of healthcare services, by using the existing medical information, without being necessary to investigate again the patient in another MU if this has been already investigated elsewhere.

\section{References}

[1] Regulation (EU) 2016/679 of the European Parliament and of the Council of 27 April 2016 on the protection of natural persons with regard to the processing of personal data and on the free movement of such data, and repealing Directive 95/46/EC (General Data Protection Regulation)

[2] M.E. Rac-Albu, V. Ciobanu, M. Rac-Albu, N. Popescu (2016) ,Interoperability of Medical Data Through e-Health Service in Romania"(IESS 2016), The 7Th International Workshop on Exploring Service Science, edited by Borangiu $\mathrm{T}$, Dragoicea M, Novoa H, Bucharest, Univ. Politehnica Bucharest. Fac Automat 
Control \& Computer Science, 25-27 May 2016, Volum 247, pp. 683-692, I SSN 1865-1348, ISNN (electronic) 1865-1356.

[3] C. Lubamba, A. Bagula,(2017)"Cyberhealthcare cloud computing interoperability using HL7-CDA standard" -2017 IEEE Symposium on Computer and Communication

[4] A. Milstein „Moving past the EHR interoperability blame game", J NEJM Catalyst. Feb 22, 2017.

[5] HIMSS Dictionary of Healthcare Information Technology Terms, Acronyms and Organizations, 2nd Edition, 2010, http://www.himss.org/library/interoperabi lity-standards/what-is-interoperability

[6] H. Vernon Anderson, William S. Weintraub, Martha J. Radford, Mark S. Kremers, Matthew T. Roe, Richard E. Shaw, Dana M. Pinchotti and James E. Standardized Cardiovascular Data for Clinical Research, Registries, and Patient Care: A Report From the Data Standards Workgroup of the National Cardiovascular Research Infrastructure Project, Tcheng, Journal of the American College of Cardiology, May 2013.

[7] ACCF/AHA 2011 Key Data Elements and Definitions of a Base Cardiovascular
Vocabulary for Electronic Health Records. A Report of the American College of Cardiology Foundation/American Heart Association Task Force on Clinical Data Standards, Circulation. 2011; 124:103-123, Originally published July 5, 2011

[8] N. C. Chheda. Standardization \& Certification: The Truth Just Sounds Different. Report, 2007

[9] B. Walker, J. Pan, et al.: The Value of Health Care Information Exchange and Interoperability. Health Affairs, pp. 10-18, 2005.

[10] S. Bhartiyaa, D. Mehrotraa, A. Girdhar: Issues in Achieving Complete Interoperability while Sharing Electronic Health Records. Procedia Computer Science,Volume 78, 2016, Pages 192-198

[11]HL7ClinicalDocumentArchitecture, http: //www.hl7.org/implement/standards/prod uct_brief.cfm?product_id=7

[12] E. Charles Kahn, Jr, MD, MS, Curtis P. Langlotz, MD, PhD, David S. Channin, MD, and Daniel L. Rubin, MD, MS: Informatics in Radiology: An Information Model of the DICOM Standard1, Radiographics. 2011 Jan-Feb;

[13] https://www.lucidchart.com/pages/bpmn

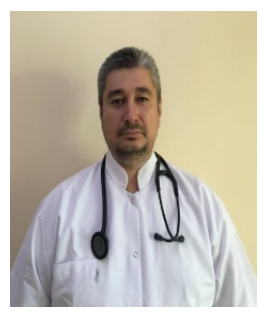

Marius RAC-ALBU (44 years) is lecturer at "Carol Davila" University of Medicine and Pharmacy, Faculty of Medicine in Bucharest, Department of Medical Informatics and Biostatistics. He graduated from University in 2000 and in 2005 becomes professor assistant at the Department of Medical Informatics and Biostatistics. In 2009 he obtained the title of specialist epidemiologist, and in 2016 he became specialist in cardiology. In 2014 he became $\mathrm{PhD}$ in medicine. His main research interests are: telemedicine, interoperability of medical data, the ambulatory monitoring of patients with heart rhythm disorders, cardiac rehabilitation patients with cardiac pathology.

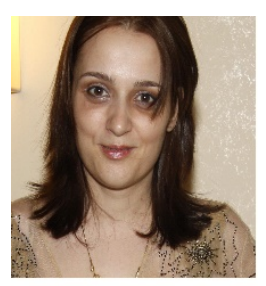

Mădălina Elena RAC-ALBU (43 years) is professor assistant at „Carol Davila" University of Medicine and Pharmacy in Bucharest Faculty of Medicine, Department of Medical Informatics and Biostatistics. She graduated the Bucharest Academy of Economic Studies in 1998 and now she is $\mathrm{PhD}$ student at Bucharest Academy of Economic Studies in Economic Informatics. The topic of the research study is Interoperability of medical data - finding new ways to apply interoperability of health records in private medicine in Romania and interoperability of health information between Romania and EU (European Union) countries. 